\title{
Oophoropexy for ovarian torsion: a new easier technique
}

\author{
Tamer A. Hosny
}

\begin{abstract}
Background: Oophoropexy for ovarian torsion is easy to be done by many tools either suturing to the lateral pelvic wall, plication of the ovarian ligament or even fixation to the back of the uterus, but it is little bit difficult to do it for pregnant women with less manipulation.

Objective: We propose that using trocar site closure needle can be easier and faster technique to do this. To assess the feasibility of using the trocar site closure needle to do oophoropexy in ovarian torsion and its possible applicability.
\end{abstract}

Patients: Seven patients presented with ovarian torsion; four of them were pregnant at 7, 15, 19 and 20 weeks of gestation, two patients with ovarian hyperstimulation in IVF cycles and one adolescent patient with hemorrhagic cyst. They were diagnosed by clinical presentation and ultrasound with Doppler analysis, and confirmed by laparoscopy where they underwent detorsion and fixation of the ovary using the trocar site closure needle.

Results: Follow up of all the cases after one week showed improvement of the symptoms and normal Doppler flow of the target ovary then after three weeks by ultrasonography which revealed normal Doppler flow in the previously torsioned ovary. Two pregnant women underwent cesarean delivery where the operated ovary was observed during the delivery and was normal in shape and freely mobile with no adhesions.

Conclusion: We propose that this technique is easier, faster and more comfortable especially in ovarian torsion in pregnant women and torsion in hyperstimulated ovaries.

Keywords: Ovarian torsion, Oophoropexy, Laparoscopy, Ovarian hyperstimulation syndrome, Trocar site closure needle

\section{Background}

Ovarian torsion occurs when the ovary rotates around the infundibulopelvic ligament and the ovarian ligament interfering with its blood supply, which may be partial or complete. It is one of the most common gynecologic emergencies in all age groups [1]. The primary risk factor for the ovarian torsion is the presence of a mass which may be either a physiologic cyst or a neoplasm [2-4].

The frequent presenting symptoms are acute onset of pelvic pain, nausea, vomiting, fever, and adnexal mass with or without abnormal genital tract bleeding $[4,5]$. A high index of suspicion is required to make the

Correspondence: dr_tamer_hosny@yahoo.com

Department of Obstetrics and Gynecology, Alexandria University Hospital, 16A Mohamed Said Pasha street, San Stefano, Alexandria 21411, Egypt diagnosis especially if there is a history of ovulation induction for treatment of infertility [6] or during pregnancy. Pelvic ultrasound is still the first-line image study for diagnosing a patient with suspected ovarian torsion. The sonographic findings that are associated with ovarian torsion are described in many studies [7, 8]. Diminished or absent ovarian vessel flow on two-dimensional, color, and three-dimensional Doppler ultrasound has been proposed as a test for ovarian torsion [9-12]. Direct visualization of the rotated ovary remains the confirmatory way to diagnose the torsion, and the laparoscopic approach is typically used also to evaluate the ovarian viability [13].

Ovarian conservation is the preferred approach for premenopausal women, and most ovaries should be considered potentially viable unless there is a high degree of certainty that the ovary is not viable due to 


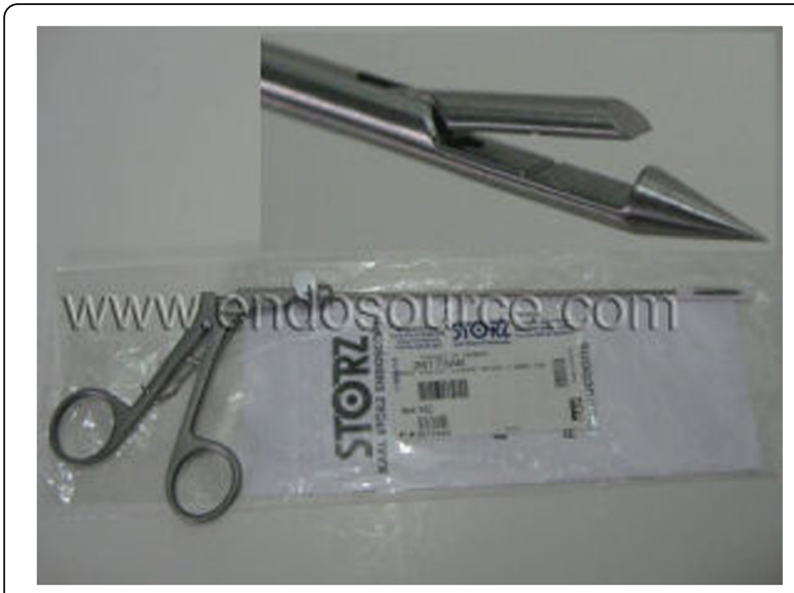

Fig. 1 Closure site trocar needle

the presence of necrotic tissue. The conservative management consists of detorsion of the ovary followed by cystectomy if a mass is present. As ovarian torsion may recur after detorsion [14, 15], unilateral or bilateral oophoropexy following detorsion may be performed to prevent recurrence [16].

\section{Patients and methods}

Seven patients presented with unilateral ovarian torsion to the emergency room in Alexandria University Hospital between November 2014 and May 2015; four of them were pregnant at 7, 15, 19, and 20 weeks of gestation; the torsioned ovaries were hyperstimulated in those pregnant women at 7, 15, and 20 weeks of gestation while there was an ovarian cyst in the pregnant woman at 19 weeks of gestation. Two patients had ovarian hyperstimulation in IVF cycles and one adolescent patient had a hemorrhagic cyst. They were diagnosed by clinical presentation and ultrasound with Doppler analysis and confirmed by conventional laparoscopy (Additional file 1: Video 1), where they underwent detorsion and fixation of the ovary using the trocar site closure needle (Fig. 1) at the same setting.

\section{Technique}

This idea is mainly to present an easy technique for emergency procedure.

$\triangleright$ Laparoscopic entry after pneumoperitoneum insufflation via Veress needle at the umbilicus or Palmer point for the pregnant women. The camera

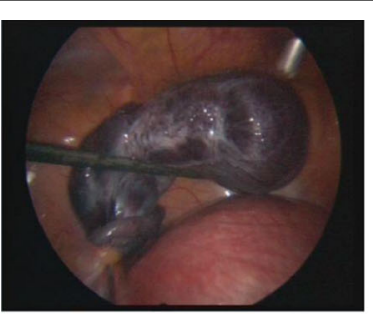

(a)

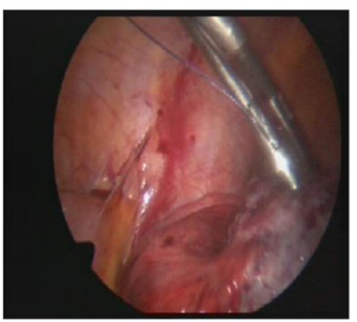

(d)

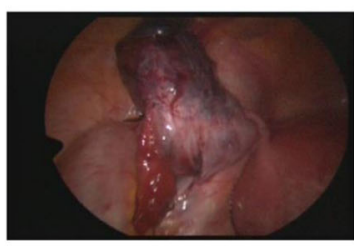

(g)

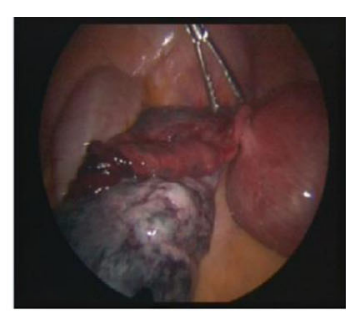

(b)

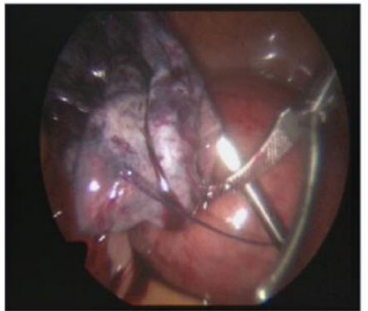

(e)

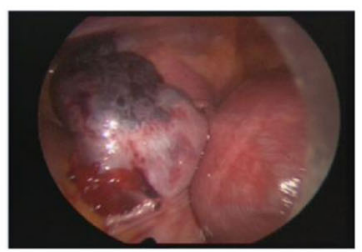

(h)

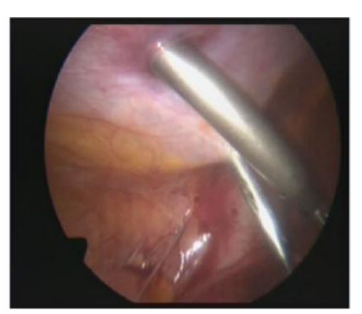

(c)

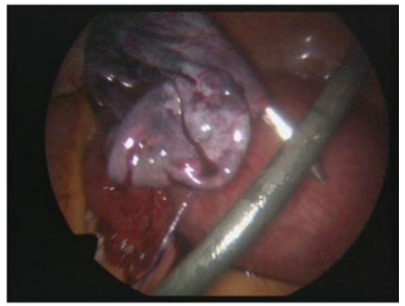

(f)

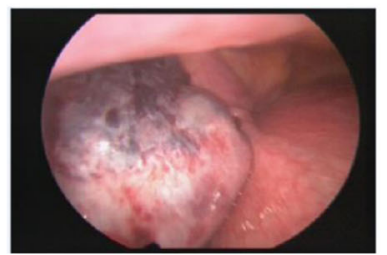

(i)

Fig. $\mathbf{2}$ a Twisted right ovary. b Detorsion. c Site of entrance for trocar site needle. $\mathbf{d}$ Entry through the ovary. e Holding threads after transfixing the ovary. $\mathbf{f}$ Second entry. $\mathbf{g}$ Fixing the ovary to the abdominal wall. $\mathbf{h}$ During deflation. i After complete deflation 
was placed in a 10-mm trocar at the umbilicus or in a 5-mm trocar at the Palmer point for the pregnant women at 19 and 20 weeks of gestation.

$\triangleright$ Using two ancillary trocars, detorsion was performed followed by ovarian bivalving or cystectomy in cases of ovarian cysts

$\triangleright$ Fixation of the ovary by transfixing the trocar site closure needle with absorbable vicryl 2-0 suture through the ovary then picking the suture from another transfixing point through the ovary then tying the suture out around the sheath (Additional file 2: Video 2).

$\triangleright$ The technique is illustrated in Fig. 2.

\section{Results}

Follow-up of all the cases after 1 week showed improvement of the symptoms and then normal Doppler flow of the target ovary after 3 weeks by ultrasonography which revealed normal Doppler flow in the previously torsioned ovary. Two pregnant women underwent cesarean delivery where the operated ovary was observed during the delivery and was normal in shape and freely mobile with no adhesions (Fig. 3).

\section{Discussion}

Although oophoropexy for ovarian torsion is debatable situation, retorsion may occur $[14,15]$. Oophoropexy for

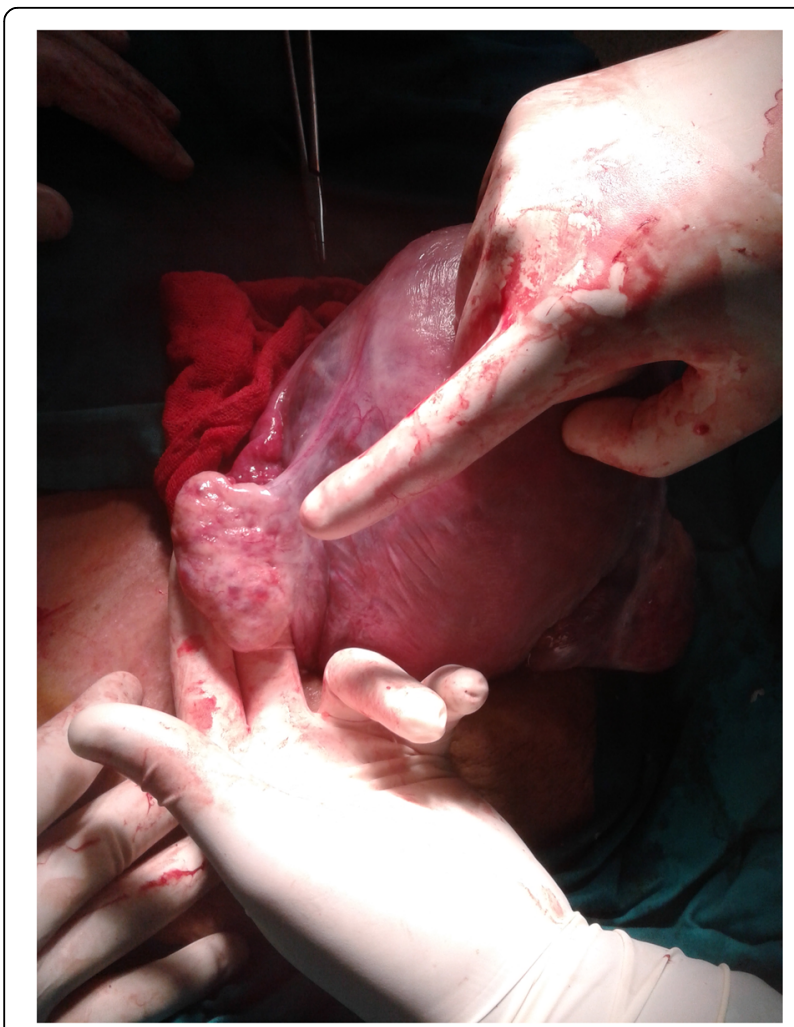

Fig. 3 Picture of ovary during cesarean section ovarian torsion is emergency procedure, if we compare the most accepted way of oophoropexy by ovarian ligament placation I think it needs more training for suturing by laparscopy and it will be very difficult in cases of pregnant uterus, so we propose that this technique may be helpful although comparative study must be done between ovarian ligament placation and this technique illustrated, but limited number of cases of ovarian torsion, as it is one of the rare emergency situation.

\section{Conclusions}

We propose that this technique is easier, faster, and more comfortable especially in ovarian torsion in pregnant women and torsion in hyperstimulated ovaries.

\section{Additional files}

Additional file 1: Video S1. (WMV $27.6 \mathrm{mb}$ )

Additional file 2: Video S2. (WMV $32.6 \mathrm{mb}$ )

\section{Competing interests}

The author declares that he has no competing interests.

Ethics approval and consent to participate

All procedures followed were in accordance with the ethical standards of the responsible committee (Alexandria university ethics committee) on human experimentation (institutional and national) and with the Helsinki Declaration of 1975, as revised in 2008 informed consent taken from all patients.

\section{Publisher's Note}

Springer Nature remains neutral with regard to jurisdictional claims in published maps and institutional affiliations.

Received: 1 February 2017 Accepted: 23 March 2017

Published online: 09 May 2017

\section{References}

1. McWilliams GD, Hill MJ, Dietrich CS 3rd (2008) Gynecologic emergencies. Surg Clin North Am 88:265

2. Varras M, Tsikini A, Polyzos D et al (2004) Uterine adnexal torsion: pathologic and gray-scale ultrasonographic findings. Clin Exp Obstet Gynecol 31:34

3. Houry D, Abbott JT (2001) Ovarian torsion: a fifteen-year review. Ann Emerg Med 38:156

4. White M, Stella J (2005) Ovarian torsion: 10-year perspective. Emerg Med Australas 17:231

5. Huchon C, Panel P, Kayem G et al (2012) Does this woman have adnexal torsion? Hum Reprod 27:2359

6. Gorkemli H, Camus M, Clasen K (2002) Adnexal torsion after gonadotrophin ovulation induction for IVF or ICSI and its conservative treatment. Arch Gynecol Obstet 267:4

7. Anthony EY, Caserta MP, Singh J, Chen MY (2012) Adnexal masses in female pediatric patients. AJR Am J Roentgenol 198:W426

8. Wilkinson C, Sanderson A (2012) Adnexal torsion-a multimodality imaging review. Clin Radiol 67:476

9. Albayram F, Hamper UM (2001) Ovarian and adnexal torsion: spectrum of sonographic findings with pathologic correlation. J Ultrasound Med 20:1083

10. Vijayaraghavan SB (2004) Sonographic whirlpool sign in ovarian torsion. J Ultrasound Med 23:1643

11. Yaman C, Ebner T, Jesacher K (2002) Three-dimensional power Doppler in the diagnosis of ovarian torsion. Ultrasound Obstet Gynecol 20:513

12. Lee EJ, Kwon HC, Joo HJ et al (1998) Diagnosis of ovarian torsion with color Doppler sonography: depiction of twisted vascular pedicle. J Ultrasound Med 17:83 
13. Oelsner G, Cohen SB, Soriano D et al (2003) Minimal surgery for the twisted ischaemic adnexa can preserve ovarian function. Hum Reprod 18:2599

14. Pansky M, Smorgick N, Herman A et al (2007) Torsion of normal adnexa in postmenarchal women and risk of recurrence. Obstet Gynecol 109:355

15. Grunewald B, Keating J, Brown S (1993) Asynchronous ovarian torsion-the case for prophylactic oophoropexy. Postgrad Med J 69:318

16. Abeş M, Sarihan H (2004) Oophoropexy in children with ovarian torsion. Eur J Pediatr Surg 14:168

\section{Submit your manuscript to a SpringerOpen ${ }^{\circ}$ journal and benefit from:}

- Convenient online submission

- Rigorous peer review

- Immediate publication on acceptance

- Open access: articles freely available online

- High visibility within the field

- Retaining the copyright to your article

Submit your next manuscript at $>$ springeropen.com 